\title{
Geociências
}

\section{Diamantes do médio rio Jequitinhonha, Minas Gerais: qualificação gemológica e análise granulométrica}

\author{
Mario Luiz de Sá C. Chaves \\ Departamento de Geologia - IGC/UFMG, Belo Horizonte \\ E-mail:mchaves@igc.ufmg.br \\ Luís Chambel \\ Instituto Superior Técnico e Sinese Consultoria, Lisboa (Portugal) \\ E-mail: luischambel@sinese.pt
}

\section{Resumo}

Os depósitos aluvionares da bacia do Rio Jequitinhonha, em Minas Gerais, constituíram a fonte da maior parte dos diamantes produzidos no Brasil desde 1714 até meados da década de 1980. Essa importância histórica e econômica motivou a apresentação dos dados quanto à granulometria e qualificação gemológica dos diamantes nas áreas de concessão das mineradoras Tejucana e Rio Novo. Em adição, a amostragem adquirida em 14 pontos ao longo do rio é instrumental para a composição de um banco de dados, tendo em vista a identificação da origem de populações de diamantes. No mega-lote estudado, constituído por 186.052 pedras $(17.689 \mathrm{ct})$, merece ser destacada a grande proporção $(82,2 \%)$ de diamantes gemológicos.

Palavras-chave: Rio Jequitinhonha, diamante, distribuição granulométrica, qualidade gemológica.

\begin{abstract}
The Jequitinhonha River basin alluvial deposits, in Minas Gerais, were the source of most of the Brazilian diamond production since 1714 until the last middle eighties. This historical and economical importance is in itself a reason to publish grain-size and gemological quality data concerning the diamonds of the Tejucana and Rio Novo mining companies concession areas. In addition, extensive sampling (186,052 stones or 17,689 ct) on 14 locations along the river can contribute to create an important database to identify the origin of different diamond populations. Among other observations, the high proportion $(82,2 \%)$ of gem diamonds should be stressed.
\end{abstract}

Keywords: Jequitinhonha River, diamond, grain-size distribution, gemological quality. 


\section{Introdução}

Diamantes foram descobertos no Brasil nas proximidades de Diamantina, centro-norte de Minas Gerais, ao início do século XVIII. Nesse contexto, a bacia hidrográfica do rio Jequitinhonha se destaca por sua importância, não só histórica, como também comercial, uma vez que a maior parte dos diamantes daquele distrito foram produzidos sobre tal bacia, nas suas porções superior e média. No médio curso do rio Jequitinhonha, os aluviões são mais largos, permitindo a operação de grandes dragas de alcatruzes, como as das mineradoras Tejucana e Rio Novo, ao contrário do que ocorre no seu alto curso. O objetivo do presente trabalho é apresentar os dados quanto a granulometria e qualidade comercial referentes à produção de diamantes do Médio Jequitinhonha. Além disso, busca-se compor um banco de dados que apóie o desenvolvimento de um modelo para a identificação da origem de diferentes populações de diamantes.

\section{Depósitos diamantíferos do rio Jequitinhonha}

Na porção superior do rio Jequitinhonha, os vales são apertados, freqüentemente formando canyons entalhados sobre as rochas quartzíticas da serra do Espinhaço. Nessa área, como a largura dos aluviões raramente excede os $20 \mathrm{~m}$, somente atividades garimpeiras são viáveis. A partir da localidade de Mendanha (Figura 1), o rio ganha o seu médio curso, desenvolvendo aluviões mais largos, muitas vezes com oflat alcançando $1.000 \mathrm{~m}$ de largura, onde as companhias Tejucana (atualmente com os serviços interrompidos) e Rio Novo operam diversas dragas de alcatruzes, acompanhadas, respectivamente, de dragas de sucção (Figura 2). No processo minerador, a draga de sucção segue à frente retirando o capeamento arenoso, estéril, enquanto a draga de alcatruzes, em seguida, escava, recolhe e trata o cascalho basal do depósito, rico em diamantes (ouro também é recuperado como subproduto).
A lavra de diamantes aluvionares do rio Jequitinhonha abrange exclusivamente sua calha atual, de idade recente a sub-recente. A fonte desses diamantes está concentrada nos conglomerados proterozóicos intercalados na Formação Sopa-Brumadinho, aflorantes em porções altas da serra do Espinhaço nas cabeceiras do rio e sua margem oeste (Figura 1), constituindo, assim, um novo

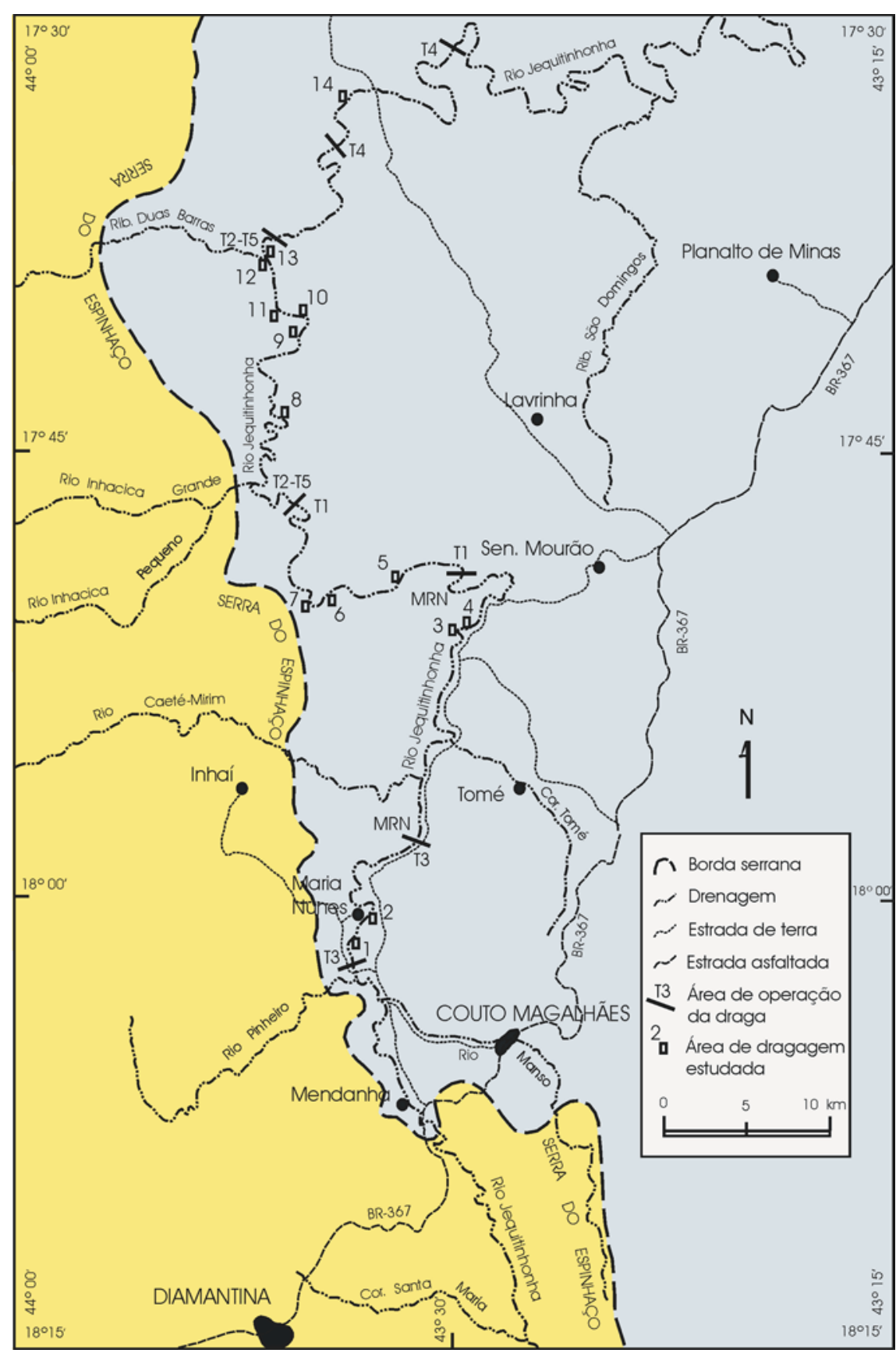

Figura 1 - Mapa da região do rio Jequitinhonha ao norte de Diamantina (MG), ressaltando as quatorze áreas de dragagem das mineradoras Tejucana e Rio Novo, onde lotes de diamantes foram examinados no presente estudo. 
dia do cascalho mineralizado é de $4 \mathrm{~m}$, para uma cobertura estéril que, em geral, alcança porte similar (Figura 3-B).

\section{Identificação da fonte de lotes de diamantes}

Desde quando foi percebido que a produção diamantífera de certos países africanos, como Angola, Serra Leoa e Congo, estava atrelada ao financiamento de grupos engajados em guerras civis locais (os chamados conflict diamonds, também conhecidos em português como "diamantes-de-sangue"), uma campanha internacional patrocinada pela ONU tem procurado impor sanções à importação de material desses países. Além disso, a comunidade consumidora, sentindo-se moralmente abalada por tais acontecimentos, estimulou a pesquisa de propostas científicas visando a conhecer a real procedência dos lotes de diamantes, para evitar que essa produção chegasse aos grandes centros lapidadores. Entretanto, logo ficou claro que inexistiam metodologias científicas seguras capazes de identificar tal procedência (Janse, 2000; Shigley, 2002).

Desde longa data se tem percebido que diferentes depósitos diamantíferos, desde os primários, mostram particularidades específicas (Lewis, 1887). Nesse sentido, as médias de tamanho, valor ou qualidade gemológica, a freqüência relativa de formas cristalográficas, a presença de certas variedades, bem como outras propriedades químicas afins, poderiam ser relacionados com certos depósitos ou áreas diamantíferas. Estudos nesse sentido foram inicialmente propostos para alguns kimberlitos sul-africanos (Harris et al., 1975, 1979), norte-americanos (Otter et al., 1994) e para os pláceres costeiros da Namíbia (Sutherland, 1982). No Brasil, estudos semelhantes incluíram os diamantes da mina de Romaria Triângulo Mineiro (Svisero \& Haralyi, 1985), do rio Tibagi - Paraná (Chieregatti, 1989) e da serra do Espinhaço - norte de Minas Gerais (Chaves, 1997; Chaves et al., 1998).

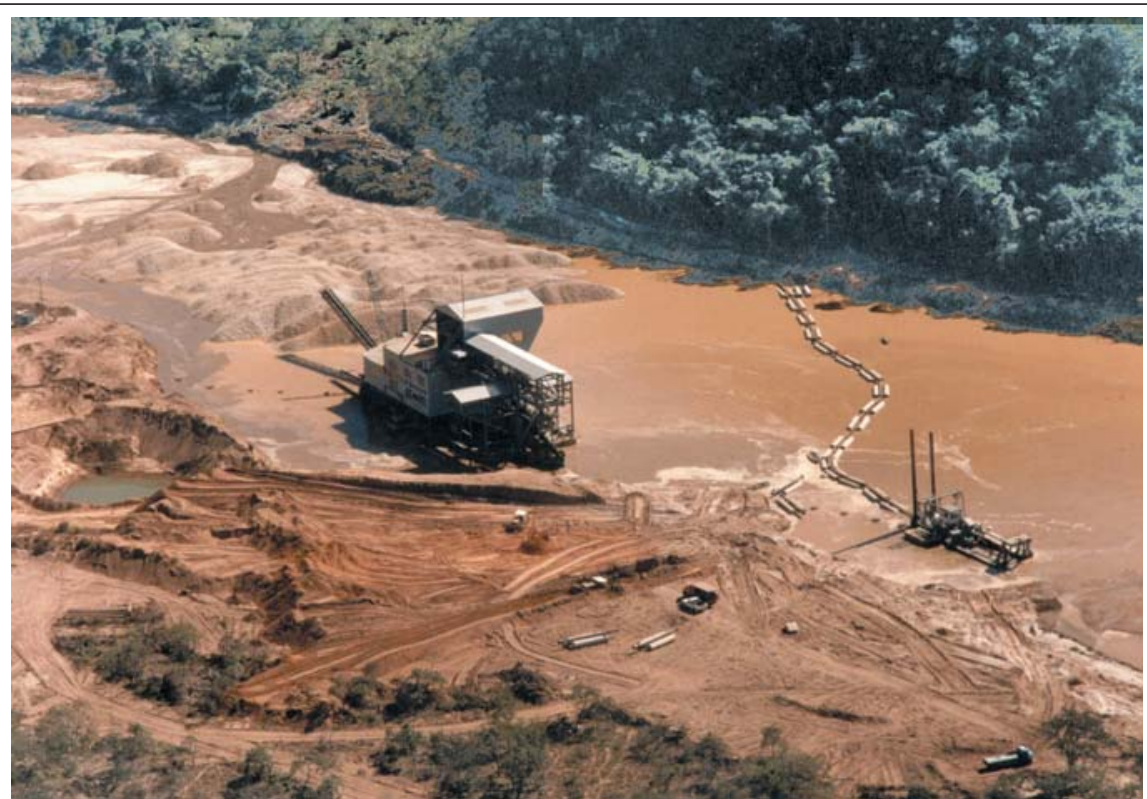

Figura 2 - Uma das dragas de alcatruzes em operação no depósito aluvionar do médio rio Jequitinhonha ("Maria Bonita", à esquerda), tendo a frente (à direita) a draga de sucção do recobrimento arenoso estéril (foto cedida pela Mineração Rio Novo).

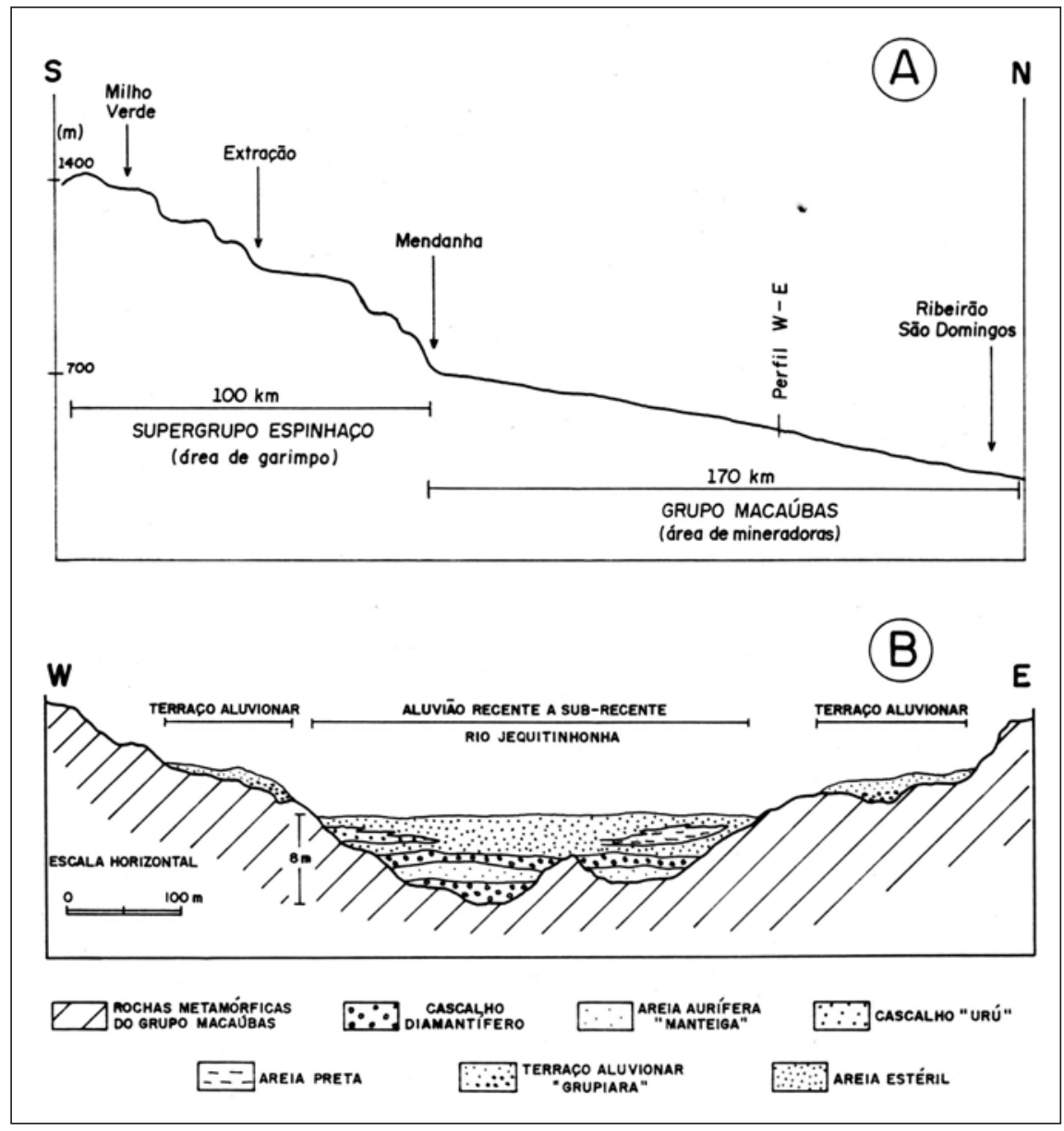

Figura 3 - (A) Perfil topográfico esquemático mostrando o rio Jequitinhonha desde o seu alto curso nas proximidades de Milho Verde (Serro), até a queda brusca de seu gradiente e jusante de Mendanha. (B) Seção ideal, transversal à calha do rio em seu médio curso, na área de lavra da Mineração Rio Novo (modificada de Chaves \& Uhlein, 1991). 
Diversos autores (Chambel, 2000a,b; Chaves et al., 1998; Janse, 2000; Shigley, 2002) procuraram enfatizar que os diamantes de determinado depósito têm uma história geológica comum e, assim, devem possuir características que são "únicas" para cada depósito. Documentando tais características, elas poderiam conduzir à identificação do local de origem do lote de diamantes. Para isso, entretanto, precisa-se envolver análises estatísticas sobre populações de diamantes com grande número de indivíduos e os resultados precisam de ser compilados dentro de um programa de dados para cada área produtora de diamantes do mundo. Tal assinatura mineralógica, ainda que bastante fácil de se obter nas jazidas em fontes primárias, torna-se mais complicada em relação aos depósitos secundários, muitas vezes dispersos sobre grandes regiões. A apresentação dos dados referentes aos aluviões do rio Jequitinhonha pretende ser uma contribuição a tal proposta.

\section{Discussão dos dados}

$\mathrm{Na}$ área da Cia. Tejucana, os dados utilizados, no presente estudo, compõem-se de 14 parcelas correspondendo à produção mensal de cinco dragas (T1-T5), quando em plena operação nas décadas de 1980-90 (Figura 1, Tabela 1). Tal produção foi classificada originalmente pelos técnicos dessa companhia em termos granulométricos e comerciais, nos quatro grupos principais: (1) diaman- tes gemológicos de $1^{a}$ qualidade, (2) diamantes gemológicos de $2^{\mathrm{a}}$ qualidade, (3) chips e (4) diamantes industriais. Os chips correspondem a diamantes de qualidade gemológica inferior, por apresentarem cristalização irregular ou geminada (Chaves \& Chambel, 2003). Nesse trabalho, os diamantes de melhor qualidade $\left(1^{\mathrm{a}} / 2^{\mathrm{a}}\right)$ foram agrupados constituindo os diamantes "gemas", conforme referido nas Tabelas 3, 4 e 5.

Em relação aos estudos realizados na área de concessão da Mineração Rio Novo, somente duas amostragens foram utilizadas (janeiro e junho/1994), referentes a cerca da metade da produção mensal em porções distintas do setor de lavra conhecido como "Lagoa Seca" (jusante e montante), um distando do ou-

Tabela 1 - Sumário dos dados de amostragens nas dragas das companhias Tejucana e Rio Novo (rio Jequitinhonha), organizados de montante para jusante (pontos localizados na Figura 1).

\begin{tabular}{|c|c|c|c|c|c|c|}
\hline Ponto & Draga & $\begin{array}{l}\text { Época da } \\
\text { Lavra }\end{array}$ & $\begin{array}{l}\text { Produção } \\
\text { (ct) }\end{array}$ & $\begin{array}{l}\text { Produção } \\
\text { (nº pedras) }\end{array}$ & $\begin{array}{c}N^{0} \text { pedras/ct } \\
(p / c t)\end{array}$ & $\begin{array}{c}\text { Média por trecho } \\
\text { (p/ct) }\end{array}$ \\
\hline 1 & T3 & Fev/85 & 306 & 818 & 2,67 & \multirow{2}{*}{5,17} \\
\hline 2 & T3 & $\mathrm{Jul} / 83$ & 455 & 3.495 & 7,68 & \\
\hline 3 & $\mathrm{M}^{\mathrm{a}}$ Bonita & Jun/94 & 1.398 & 6.931 & 4,96 & \multirow{2}{*}{6,64} \\
\hline 4 & $\mathrm{M}^{\mathrm{a}}$ Bonita & Jan/94 & 2.217 & 18.446 & 8,32 & \\
\hline 5 & T1 & Fev/82 & 2.926 & 34.153 & 11,67 & \multirow{3}{*}{9,27} \\
\hline 6 & T1 & Fev/87 & 271 & 1.403 & 5,18 & \\
\hline 7 & T1 & Ago/87 & 94 & 1.031 & 10,97 & \\
\hline 8 & T5 & Abr/89 & 2.576 & 45.184 & 17,54 & \multirow{6}{*}{12,15} \\
\hline 9 & T2 & Dez/81 & 1.326 & 11.358 & 8,56 & \\
\hline 10 & T2 & Jun/82 & 1.311 & 8.460 & 6,45 & \\
\hline 11 & $\mathrm{~T} 2$ & Jan/83 & 671 & 3.495 & 5,21 & \\
\hline 12 & $\mathrm{~T} 2$ & Out/84 & 737 & 3.686 & 5,00 & \\
\hline 13 & $\mathrm{~T} 2$ & $\mathrm{Abr} / 84$ & 1.694 & 14.535 & 8,58 & \\
\hline 14 & $\mathrm{~T} 4$ & Jun/81 & 1.707 & 33.057 & 19,36 & \multirow{2}{*}{19,36} \\
\hline \multicolumn{3}{|c|}{ Total } & 17.689 & 186.052 & & \\
\hline
\end{tabular}


Mario Luiz de Sá C. Chaves et al.

Tabela 2 - Dados sobre a granulometria (em percentagem) dos diamantes, em função do peso e do número de pedras, produzidos nas dragas em operação no médio rio Jequitinhonha.

Peso em quilates (\%)

\begin{tabular}{l|c|c|c|c|c|c|c|c|c|c|c|c|c|c}
$\begin{array}{c}\text { Ponto } \\
\text { Crivo }\end{array}$ & 1 & 2 & 3 & 4 & 5 & 6 & 7 & 8 & 9 & 10 & 11 & 12 & 13 & 14 \\
\hline $\mathbf{- 6}$ & 0,3 & 4,7 & 0,5 & 8,5 & 8,0 & 1,7 & 7,8 & 14,7 & 4,9 & 3,2 & 5,5 & 1,3 & 14,1 & 16,0 \\
\hline $\mathbf{+ 6}-\mathbf{1 0}$ & 5,4 & 20,5 & 19,8 & 16,6 & 34,1 & 17,2 & 27,7 & 45,0 & 27,7 & 21,0 & 18,1 & 18,3 & 22,0 & 55,0 \\
\hline $\mathbf{+ 1 0}-\mathbf{1 2}$ & 3,3 & 6,5 & 16,8 & 12,1 & 8,8 & 9,2 & 9,3 & 6,5 & 8,8 & 8,6 & 27,0 & 8,0 & 18,8 & 7,6 \\
\hline $\mathbf{+ 1 2}-\mathbf{1 9}$ & 51,7 & 42,2 & 42,3 & 50,0 & 33,6 & 42,9 & 36,0 & 23,7 & 34,1 & 35,7 & 23,2 & 46,8 & 29,8 & 18,2 \\
\hline $\mathbf{+ 1 9}-\mathbf{2 2}$ & 9,2 & 6,0 & 9,1 & 5,9 & 3,8 & 5,7 & 4,2 & 1,9 & 4,9 & 5,6 & 12,7 & 6,5 & 4,8 & 0,9 \\
\hline $\mathbf{+ 2 2}$ & 30,1 & 20,1 & 11,5 & 6,9 & 11,7 & 23,3 & 15,1 & 8,2 & 19,6 & 25,9 & 13,6 & 19,1 & 10,4 & 2,3 \\
\hline Total & \multicolumn{10}{|c|}{100} \\
\hline
\end{tabular}

Número de pedras (\%)

\begin{tabular}{c|c|c|c|c|c|c|c|c|c|c|c|c|c|c}
$\begin{array}{c}\text { Ponto } \\
\text { Crivo }\end{array}$ & 1 & 2 & 3 & 4 & 5 & 6 & 7 & 8 & 9 & 10 & 11 & 12 & 13 & 14 \\
\hline $\mathbf{- 6}$ & 10,5 & 39,6 & 2,1 & 43,6 & 43,6 & 20,5 & 47,1 & 54,2 & 35,8 & 31,3 & 37,3 & 15,7 & 27,9 & 50,2 \\
\hline $\mathbf{+ 6}-\mathbf{1 0}$ & 20,6 & 36,7 & 48,0 & 28,3 & 41,4 & 39,6 & 35,8 & 38,6 & 43,4 & 40,7 & 25,7 & 42,5 & 49,3 & 43,9 \\
\hline $\mathbf{+ 1 0}-\mathbf{1 2}$ & 7,2 & 5,2 & 20,2 & 8,8 & 4,6 & 11,0 & 5,2 & 2,3 & 6,2 & 8,2 & 22,6 & 9,4 & 7,6 & 2,5 \\
\hline $\mathbf{+ 1 2}-\mathbf{1 9}$ & 50,0 & 15,6 & 26,0 & 17,6 & 9,2 & 24,5 & 10,5 & 4,4 & 12,2 & 16,1 & 9,2 & 27,9 & 13,5 & 3,3 \\
\hline $\mathbf{+ 1 9}-\mathbf{2 2}$ & 4,4 & 1,0 & 2,3 & 0,9 & 0,4 & 1,4 & 0,5 & 0,2 & 0,8 & 1,1 & 4,0 & 1,7 & 0,6 & 0,1 \\
\hline $\mathbf{+ 2 2}$ & 7,3 & 1,9 & 1,4 & 0,8 & 0,7 & 3,0 & 1,0 & 0,4 & 1,5 & 2,6 & 1,1 & 2,8 & 1,0 & 0,1 \\
\hline Total & \multicolumn{10}{|c|}{100} \\
\hline
\end{tabular}

tro cerca de $1.000 \mathrm{~m}$ (Figura 1). Tal produção era proveniente de uma das duas dragas em operação pela companhia ("Maria Bonita"), pois, desde 1989, a mesma trabalhava também com a draga "Chica da Silva" (ou T1), adquirida da Mineração Tejucana. Para melhor entendimento das análises fornecidas, o trecho estudado do rio Jequitinhonha foi ainda dividido em dois setores, designados de "bloco montante" e "bloco jusante".

Há que se lamentar a falta de dados entre as localidades de Mendanha e Maria Nunes, onde os teores com certeza foram maiores por estarem logo à frente do espigão serrano (Figura 1). Sem dúvida, nesse trecho do rio Jequitinhonha, os serviços estavam concentrados na época da Coroa Portuguesa, a julgar pelos relatos de Mawe (1812) e Eschwege (1833). Com os dados fornecidos na Tabela 1, a impressão inicial é de que não existe correlação entre a distribuição das médias de peso/tamanho das pedras com o distanciamento de montante para jusante. Ainda que se verifique uma drástica diminuição desses valores desde o ponto 1 (resultando em 3 pedras para cada quilate) até $o$ ponto 14 (19 pedras/ct), nos pontos intermediários os dados apresentam-se aparentemente caóticos.

Dessa maneira, poder-se-ia, em princípio, deduzir que as distribuições granulométricas, bem como os teores em diamantes, são bastante variáveis, provavelmente em dependência do posicionamento das cabeceiras dos tributários da margem esquerda do rio (Tabela 1 - Coluna 5). Produções (e teores) maiores determinariam o quanto de superfície tal sub-bacia teria drenado áreas de afloramento do Conglomerado Sopa. Entretanto, juntando-se os dados para trechos maiores do rio, conforme a coluna 6 da mesma tabela, observa-se uma notável regularidade na diminuição das médias de tamanho das pedras, desde 5,17 pedras/quilate na área de lavra da T3 - no início do bloco montante, até 19,36 p/ct na área da T4 - ao final do bloco jusante.

Em termos de granulometria (Tabela 2), a faixa preferencial, em função do peso dos diamantes (a qual se considera como a melhor maneira de se interpretar os dados), está concentrada no crivo $[>12<19]$, a qual inclui diamantes de peso médio de 0,33 ct, com a média geral de $35,3 \%$. Interessante lembrar que tal classe, conhecida no meio comercial como 3/1 (três pedras por quilate), apresenta valores médios bastante apreciáveis de comercialização, pois ela é largamente utilizada na confecção de brilhantes de pequeno porte $(\cong 0,10 \mathrm{ct})$, os mais procurados em termos de "volume" de vendas em joalherias. 
Diamantes do médio rio Jequitinhonha, Minas Gerais: qualificação gemológica e análise granulométrica

Tabela 3 - Descrição detalhada em termos percentuais da qualidade gemológica dos diamantes em função do peso das pedras, nas dragas das minerações Tejucana (T1-T5) e Rio Novo (MRN). \%Diamantes: Gema - Gemológicos de Alta Qualidade, Chip - Chips, Ind - Industriais.

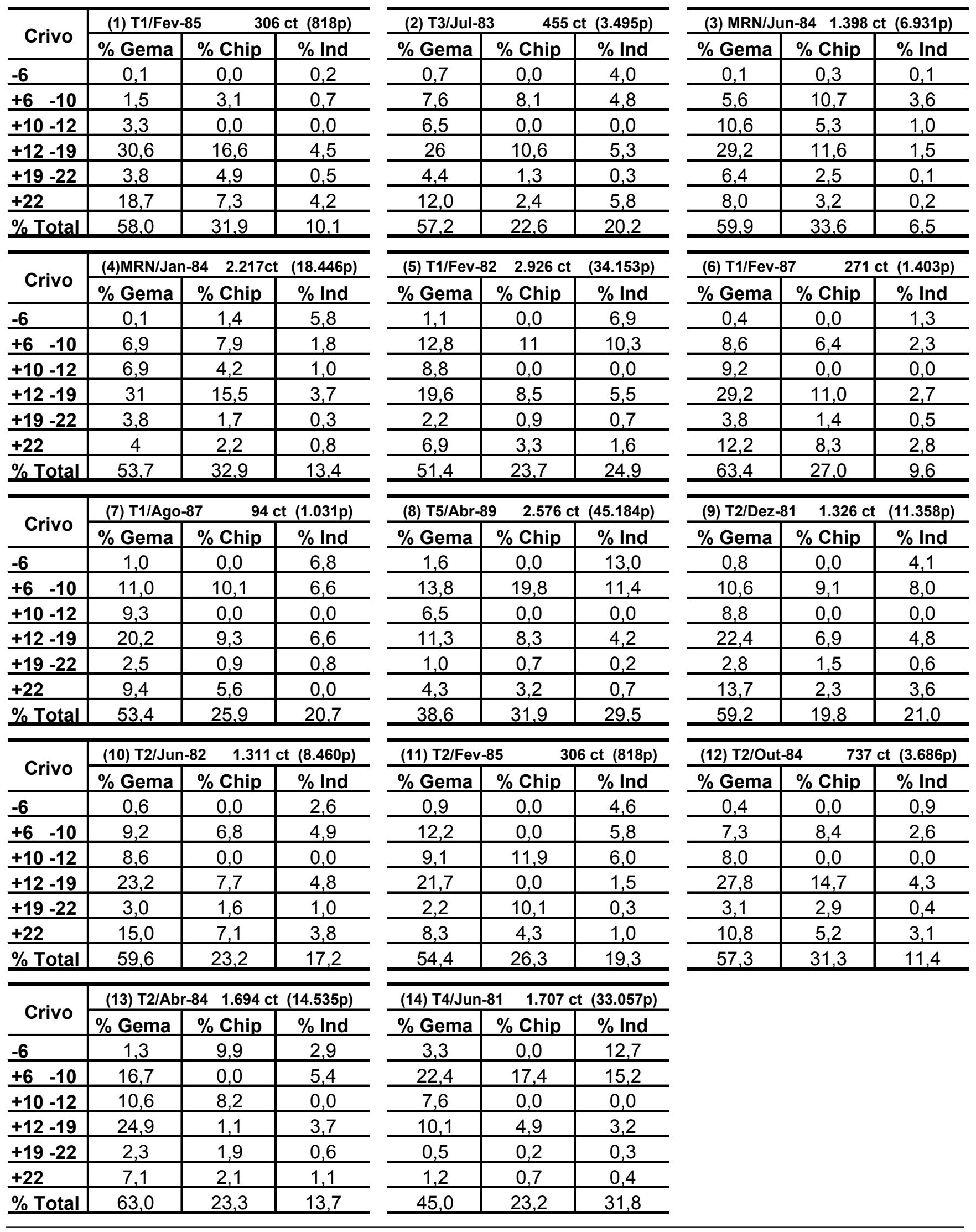


Mario Luiz de Sá C. Chaves et al.

Tabela 4 - Descrição detalhada em termos percentuais da qualidade gemológica dos diamantes em função do número de pedras, nas dragas das minerações Tejucana (T1-T5) e Rio Novo (MRN). \%Diamantes: Gema - Gemológicos de Alta Qualidade, Chip - Chips, Ind - Industriais.

\begin{tabular}{|c|c|c|c|}
\hline \multirow{2}{*}{ Crivo } & \multicolumn{2}{|c|}{ (1) T3/Fev-85 } & $818 p(306 c t)$ \\
\hline & $\%$ Cut & $\%$ Chip & $\%$ Ind \\
\hline-6 & 1,1 & 0,0 & 9,4 \\
\hline$+6-10$ & 6,7 & 11,1 & 2,7 \\
\hline$+10-12$ & 7,2 & 0,0 & 0,0 \\
\hline$+12-19$ & 28,3 & 17,6 & 4,2 \\
\hline$+19-22$ & 1,8 & 2,3 & 0,2 \\
\hline+22 & 4,3 & 1,8 & 1,2 \\
\hline$\%$ Total & 49,4 & 32,9 & 17,7 \\
\hline
\end{tabular}

\begin{tabular}{|c|c|c|c|}
\hline \multirow{2}{*}{ Crivo } & \multicolumn{3}{|c|}{ (4) MRN/Jan-94 18446p (2.217 ct) } \\
\hline & $\%$ Cut & \% Chip & $\%$ Ind \\
\hline-6 & 3,3 & 5,7 & 34,6 \\
\hline$+6 \quad-10$ & 9,7 & 15,2 & 3,4 \\
\hline$+10-12$ & 4,8 & 3,5 & 0,5 \\
\hline$+12-19$ & 10,4 & 5,8 & 1,3 \\
\hline$+19-22$ & 0,6 & 0,3 & 0,1 \\
\hline+22 & 0,5 & 0,2 & 0,1 \\
\hline$\%$ Total & 29,3 & 30,7 & 40 \\
\hline \multirow{2}{*}{ Crivo } & \multicolumn{2}{|c|}{\begin{tabular}{l|ll} 
(7) T1/Ago-87 & 1. \\
\end{tabular}} & $1.031 \mathrm{p}(94 \mathrm{ct})$ \\
\hline & $\%$ Cut & \% Chip & $\%$ Ind \\
\hline-6 & 2,8 & 0,0 & 44,3 \\
\hline$+6-10$ & 13,0 & 13,4 & 9,4 \\
\hline$+10-12$ & 5,2 & 0,0 & 0,0 \\
\hline$+12-19$ & 5,6 & 2,8 & 2,0 \\
\hline$+19-22$ & 0,3 & 0,1 & 0,1 \\
\hline+22 & 0,7 & 0,3 & 0,0 \\
\hline$\%$ Total & 27,6 & 16,6 & 55,8 \\
\hline
\end{tabular}

\begin{tabular}{|c|c|c|c|}
\hline \multirow{2}{*}{ Crivo } & (10) T2/Jun-82 & \multicolumn{2}{|c|}{$8.460 p(1.311 c t)$} \\
\hline & $\%$ Cut & \% Chip & $\%$ Ind \\
\hline-6 & 2,6 & 0,0 & 28,7 \\
\hline $\begin{array}{ll}+6 & -10 \\
\end{array}$ & 17,1 & 13,6 & 9,9 \\
\hline$+10-12$ & 8,2 & 0,0 & 0,0 \\
\hline$+12-19$ & 10,1 & 3,9 & 2,2 \\
\hline$+19-22$ & 0,6 & 0,3 & 0,2 \\
\hline+22 & 1,5 & 0,7 & 0,5 \\
\hline$\%$ Total & 40,0 & 18,5 & 41,5 \\
\hline \multirow{2}{*}{ Crivo } & \multicolumn{3}{|c|}{ (13) T2/Abr-84 14.535p (1.694 ct) } \\
\hline & $\%$ Cut & $\%$ Chip & $\%$ Ind \\
\hline-6 & 4,3 & 0,0 & 23,7 \\
\hline$+6 \quad-10$ & 25,0 & 15,8 & 8,5 \\
\hline$+10-12$ & 7,6 & 0,0 & 0,0 \\
\hline$+12-19$ & 8,6 & 3,4 & 1,5 \\
\hline$+19-22$ & 0,4 & 0,2 & 0,1 \\
\hline+22 & 0,6 & 0,3 & 0,1 \\
\hline$\%$ Total & 46,5 & 19,6 & 33,9 \\
\hline
\end{tabular}

\begin{tabular}{c|c|c}
\hline (2) T3/Jul-83 & \multicolumn{2}{c}{$3.495 p$ (455 ct) } \\
\hline \% Cut & \% Chip & \% Ind \\
\hline 2,5 & 0,0 & 37,0 \\
\hline 13,2 & 14,8 & 8,7 \\
\hline 5,2 & 0,0 & 0,0 \\
\hline 9,1 & 4,4 & 2,1 \\
\hline 0,7 & 0,2 & 0,1 \\
\hline 1,2 & 0,3 & 0,4 \\
\hline 31,9 & 19,7 & 48,4 \\
\hline
\end{tabular}

(5) T1/Fev-82 $34.153 \mathrm{p}(2.926 \mathrm{ct})$

\begin{tabular}{c|c|c}
\hline$\%$ Cut & $\%$ Chip & $\%$ Ind \\
\hline
\end{tabular}

\begin{tabular}{l|l|l|l|}
\hline 2,6 & 0,0 & 41,0 \\
\hline
\end{tabular}

\begin{tabular}{c|c|c}
\hline 14,4 & 13,7 & 13,3 \\
\hline 4,6 & 0,0 & 0,0 \\
\hline 4,9 & 2,7 & 1,7 \\
\hline 0,3 & 0,1 & 0,1 \\
\hline 0,4 & 0,2 & 0,1 \\
\hline 27,2 & 16,7 & 56,2 \\
\hline
\end{tabular}

\begin{tabular}{c|c|c}
\hline (8) T5/Abr-89 & 45.184p (2.576 ct) \\
\hline \% Cut & \% Chip & \% Ind \\
\hline 2,5 & 0,0 & 51,6 \\
\hline 10,4 & 16,4 & 11,8 \\
\hline 2,3 & 0,0 & 0,0 \\
\hline 1,8 & 1,7 & 0,9 \\
\hline 0,1 & 0,1 & 0,0 \\
\hline 0,2 & 0,1 & 0,1 \\
\hline 17,3 & 18,3 & 64,4 \\
\hline
\end{tabular}

\begin{tabular}{c|c|c}
\hline \multicolumn{2}{|c|}{ (11) T2/Jan-83 } & \multicolumn{2}{c}{ 6.236p (671 ct) } \\
\hline \% Cut & \% Chip & \% Ind \\
\hline 2,6 & 0,0 & 34,7 \\
\hline 16,4 & 0,0 & 9,4 \\
\hline 6,1 & 16,5 & 0,0 \\
\hline 7,0 & 0,0 & 2,1 \\
\hline 0,4 & 3,5 & 0,2 \\
\hline 0,6 & 0,4 & 0,1 \\
\hline 33,1 & 40,4 & 46,5 \\
\hline
\end{tabular}

(14) T4/Jun-81 33.057p (1.707 ct) \begin{tabular}{c|c|c}
\hline$\%$ Cut & $\%$ Chip & $\%$ Ind \\
\hline
\end{tabular}

\begin{tabular}{c|c|c}
\hline 4,6 & 0,0 & 45,6 \\
\hline 16,1 & 14,6 & 13,2 \\
\hline 2,5 & 0,0 & 0,0 \\
\hline 1,7 & 1,0 & 0,7 \\
\hline 0,0 & 0,0 & 0,0 \\
\hline 0,1 & 0,0 & 0,0 \\
\hline 24,9 & 15,6 & 59,5 \\
\hline
\end{tabular}

\begin{tabular}{c|c|c}
\hline \multicolumn{2}{|c|}{ (3) MRN/Jun-84 } & \multicolumn{2}{c}{ 6.931p (1.398 ct) } \\
\hline \% Cut & \% Chip & \% Ind \\
\hline 0,7 & 1,0 & 0,4 \\
\hline 14,8 & 24,5 & 8,7 \\
\hline 12,1 & 6,9 & 1,2 \\
\hline 16,5 & 8,2 & 1,3 \\
\hline 1,6 & 0,7 & 0,0 \\
\hline 1,0 & 0,4 & 0,0 \\
\hline 46,7 & 41,7 & 11,6 \\
\hline
\end{tabular}

(6) T1/Fev-87 1.403 p (271 ct)

\begin{tabular}{|c|c|c|}
\hline$\%$ Cut & $\%$ Chip & $\%$ Ind \\
\hline
\end{tabular}

\begin{tabular}{l|c|c|c|}
\hline 2,3 & 0,0 & 18,3 \\
\hline
\end{tabular}

\begin{tabular}{c|c|c}
19,8 & 13,4 & 6,3 \\
\hline
\end{tabular}

\begin{tabular}{l|l|l}
11,0 & 0,0 & 0,0 \\
\hline 15,7 & 6,8 & 2,0 \\
\hline
\end{tabular}

\begin{tabular}{l|l|l}
$11,0,7$ & 6,8 & 2,0 \\
\hline 0,9 & 0,4 & 0,1 \\
\hline
\end{tabular}

\begin{tabular}{l|l|l}
0,9 & 0,4 & 0,1 \\
\hline 1,6 & 1,0 & 0,4
\end{tabular}

1,6

51,3

1,0

0,4

\begin{tabular}{|c|c|c|}
\hline (9) T2/Dez-81 & \multicolumn{2}{|c|}{$11.358 p(1.326 c t)$} \\
\hline$\%$ Cut & \% Chip & $\%$ Ind \\
\hline 2,5 & 0,0 & 33,3 \\
\hline 16,1 & 14,2 & 13,2 \\
\hline 6,2 & 0,0 & 0,0 \\
\hline 7,4 & 2,9 & 1,9 \\
\hline 0,4 & 0,2 & 0,1 \\
\hline 1,0 & 0,2 & 0,3 \\
\hline 33,7 & 17,5 & 48,8 \\
\hline \multicolumn{2}{|c|}{ (12) T2/Out-84 } & o (737 ct) \\
\hline$\%$ Cut & \% Chip & $\%$ Ind \\
\hline 2,0 & 0,0 & 13,8 \\
\hline 17,3 & 18,6 & 6,6 \\
\hline 9,4 & 0,0 & 0,0 \\
\hline 15,5 & 9,8 & 2,5 \\
\hline 0,8 & 0,8 & 0,1 \\
\hline 1,5 & 0,8 & 0,4 \\
\hline 46,5 & 30,0 & 23,4 \\
\hline
\end{tabular}


Tabela 5 - Dados gerais sobre diamantes gemológicos, chips e industriais (em percentagem), nas dragas em operação no médio rio Jequitinhonha.

Peso em quilates (\%)

\begin{tabular}{c|c|c|c|c|c|c|c|c|c|c|c|c|c|c}
\hline $\begin{array}{c}\text { Ponto } \\
\text { Qualidade }\end{array}$ & $\mathbf{1}$ & $\mathbf{2}$ & $\mathbf{3}$ & $\mathbf{4}$ & $\mathbf{5}$ & $\mathbf{6}$ & $\mathbf{7}$ & $\mathbf{8}$ & $\mathbf{9}$ & $\mathbf{1 0}$ & $\mathbf{1 1}$ & $\mathbf{1 2}$ & $\mathbf{1 3}$ & $\mathbf{1 4}$ \\
\hline Gema & 58,0 & 57,2 & 59,9 & 53,7 & 51,4 & 63,4 & 53,4 & 38,6 & 59,2 & 59,6 & 54,5 & 57,3 & 63,0 & 45,2 \\
\hline Chip & 31,9 & 22,6 & 33,6 & 32,9 & 23,7 & 27,0 & 25,9 & 31,9 & 19,8 & 23,2 & 26,2 & 31,3 & 23,3 & 23,2 \\
\hline Industrial & 10,1 & 20,2 & 6,5 & 13,4 & 24,9 & 9,6 & 20,7 & 29,5 & 21,0 & 17,2 & 19,3 & 11,4 & 13,7 & 31,6 \\
\hline Total & \multicolumn{10}{|c|}{100,0} \\
\hline
\end{tabular}

Número de pedras (\%)

\begin{tabular}{c|c|c|c|c|c|c|c|c|c|c|c|c|c|c}
\hline $\begin{array}{c}\text { Ponto } \\
\text { Qualidade }\end{array}$ & $\mathbf{1}$ & $\mathbf{2}$ & $\mathbf{3}$ & $\mathbf{4}$ & $\mathbf{5}$ & $\mathbf{6}$ & $\mathbf{7}$ & $\mathbf{8}$ & $\mathbf{9}$ & $\mathbf{1 0}$ & $\mathbf{1 1}$ & $\mathbf{1 2}$ & $\mathbf{1 3}$ & $\mathbf{1 4}$ \\
\hline Gema & 49,4 & 31,9 & 46,7 & 29,3 & 27,2 & 51,3 & 27,6 & 17,3 & 33,7 & 40,0 & 33,1 & 46,5 & 46,5 & 24,9 \\
\hline Chip & 32,9 & 19,8 & 41,7 & 30,7 & 16,7 & 21,5 & 16,6 & 18,3 & 17,5 & 18,5 & 20,4 & 30,0 & 19,6 & 15,6 \\
\hline Industrial & 17,7 & 48,3 & 11,6 & 40,0 & 56,1 & 27,2 & 55,8 & 64,4 & 48,8 & 41,5 & 46,5 & 23,5 & 33,9 & 59,5 \\
\hline Total & \multicolumn{10}{|c|}{100,0} \\
\hline
\end{tabular}

Em relação às qualidades gemológicas dos lotes (Tabelas 3,4,5), algumas observações se destacam: (1) em função do peso, a classe de granulometria $[>12$ $<19]$ também apresenta amplo predomínio em termos de diamantes gemológicos de alta qualidade, variando entre 19,6-31,0\%, com média de $26,5 \%$ para este crivo nos sete pontos do bloco montante, e 10,1-27,8\% no bloco jusante (média de 20,2\%); (2) a média total de diamantes lapidáveis (gemas + chips) atingiu o máximo de $93,5 \%$ (Ponto 3 - bloco montante), com média geral de $82,2 \%$ sobre o "mega-lote" (todos os 14 pontos), pesando $17.689 \mathrm{ct}$, com 186.052 pedras.

\section{Considerações finais}

As mineradoras Tejucana e Rio Novo representam raríssimas excessões no cenário nacional, no sentido de operações racionais e organizadas de lavras diamantíferas. O estudo dos dados de produção dessas empresas, por conseguinte, constitui uma excelente oportunidade de se trabalhar com dados precisos e confiáveis, para uma atividade em geral desorganizada e dominada por atividades garimpeiras. Ressalte-se também o fato de que ambas as mineradoras estão com suas reservas à beira da exaustão, tornando o estudo ainda mais premente. As populações de diamantes, ora estudadas, serão ainda úteis na criação de um grande banco de dados, visando a conhecer a proveniência geográfica de lotes de diamantes através de suas características mineralógicas.

Os diamantes do Médio Jequitinhonha, assim, embora de tamanhos médios bastante reduzidos e constituírem uma parcela ínfima da produção mundial (considerando uma produção mundial de $100.000 .000 \mathrm{ct} /$ ano e a produção do rio Jequitinhonha em $100.000 \mathrm{ct} /$ ano - isto significaria $0,1 \%$ daquele montante), po- dem ser considerados bastante interessantes pelos seus conteúdos histórico e comercial. Afinal, a bacia desse rio foi por quase 160 anos, a maior produtora mundial de diamantes. Além disso, tal produção representa uma das maiores freqüências médias mundiais de diamantes gemológicos $(82,2 \%$, conforme demonstrado). Por isso, ainda atualmente a cidade de Diamantina constitui um importante pólo de comercialização de diamantes em termos internacionais.

\section{Agradecimentos}

Agradecimentos especiais são direcionados à Min. Tejucana na pessoa de seu diretor, Eng. Fernando Vieira (Diamantina), pelo acesso aos dados dessa mineradora e autorização para publicação dos mesmos, bem como à Min. Rio Novo e ao seu geólogo-chefe à época, Dr. Ronald Fleischer (Belo Horizonte), pelas facilidades e gentilezas prestadas. 
Mario Luiz de Sá C. Chaves et al.

7. Referências

\section{bibliográficas}

CHAMBEL, L. Evolução do peso dos diamantes ao longo do Rio Chicapa (Lunda, Angola). REM - Rev. Esc. Minas, v.53, p.1-7, 2000a.

CHAMBEL, L. Discussão duma estratégia para a fileira dos diamantes em Portugal. Lisboa: Instituto Superior Técnico, 2000b. 409p. (Tese de Doutoramento).

CHAVES, M.L.S.C. Geologia e mineralogia do diamante da serra do Espinhaço em Minas Gerais. São Paulo: IG-USP, 1997. 289p. (Tese de Doutoramento).

CHAVES, M.L.S.C., UHLEIN, A. Depósitos diamantíferos da região do Alto/Médio Jequitinhonha, Minas Gerais. In: SCHOBBENHAUS, C. et alii. (eds.), Principais Depósitos Minerais do Brasil. v.IV-A. Brasília: CPRM/DNPM, 1991 p.117-138.

CHAVES, M.L.S.C. \& CHAMBEL, L. Diamante: a pedra, a gema, a lenda. São Paulo: Oficina de Textos, 2003. 231p.

CHAVES, M.L.S.C. et alii. Sobre a polêmica da origem do diamante na serra do Espinhaço (Minas Gerais): um enfoque mineralógico.
Rev. Brasileira de Geociências, v.28, p.285-294, 1998.

CHIEREGATTI, L.A. Aspectos mineralógicos, genéticos e econômicos das ocorrências diamantíferas da região nordeste do Paraná e sul de São Paulo. São Paulo: IGUSP, 1989. 180p. (Tese de Mestrado).

DUPONT, H. Jazida aluvionar de diamante do rio Jequitinhonha (MG). In: SCHOBBENHAUS, C. et alii. (eds.). Principais Depósitos Minerais do Brasil, v.IV-A. Brasília: CPRM/DNPM, 1991. p.139-148.

ESCHWEGE, W.L.v. Pluto Brasiliensis. Berlin: Reimer, 1833. 622p. (Trad. de D.F. Murta, Belo Horizonte, Itatiaia/EDUSP)

HARRIS, J.W. et alii. A classification scheme for diamond and a comparative study of South African diamond characteristics. Physics and Chemistry of the Earth, v.9, p.765-783, 1975.

HARRIS, J.W. et alii. Regional and local variations in the characteristics of diamonds from some southern african kimberlites. In: BOYD, F.R., MEYER, H.O.A. (ed.), Kimberlites, diatremes and diamonds: their geology, petrology and geochemistry. Washington: Amer. Geophysical Union, 1979. p.27-41.

JANSE, A. Conflict diamonds. Mining Journal, v.334, p.430-431, 2000.
LEWIS, H.C. On a diamantiferous peridotite, and the genesis of the diamond. Geological Magazine, v.4, p.22-24, 1887.

MAWE, J. Travels in the interior of Brazil. London: Longman, Hurst, Orme \& Brown, 1812. 336p. (Trad. de S.B. Viana, Belo Horizonte, Itatiaia/EDUSP).

OTTER, M.L. et alii. A physical characterization of the Sloan (Colorado) diamonds using a comprehensive diamond description scheme. In: INTERNAT. KIMBERLITE CONF., 5, 1991. Proceedings... Araxá: CPRM, 1994. p.1550.

SHIGLEY, J. Identifying the source of gem diamonds: requirements for a certification system. Eos. Transactions os American Geophysical Union, v.83, p.S369, 2002.

SUTHERLAND, D.G. The transport and sorting of diamonds by fluvial and marine processes. Economic Geology. v.77, p.1613-1620, 1982

SVISERO, D.P., HARALYI, N.L.E Caracterização do diamante da Mina de Romaria, Minas Gerais. In: SIMP. GEOL. MINAS GERAIS, 3, 1985, Belo Horizonte. Anais... Belo Horizonte: SBG-MG, 1985, p. 140-153.

Artigo recebido em 29/04/2004 e aprovado em 28/10/2004.

\section{$* * * * * *$}

\section{REM - Revista Escola de Minas 68 anos divulgando CIÊNCIA.}

$* * * * * *$

\section{REM: a mais antiga revista técnico- científica do setor mínero-metalúrgico.}

$\star * * * * *$

www.rem.com.br

$\star * * * * *$ 